\title{
An Adaptive Tracking Problem for a Family of Retarded Time-Delay Plants
}

\author{
Ahmadreza Momeni and Amir G. Aghdam \\ Department of Electrical and Computer Engineering, Concordia University \\ Montreal, QC Canada H3G 1M8 \\ \{a_momeni, aghdam\}@ece.concordia.ca
}

\begin{abstract}
Most of the existing switching control techniques consider only finite dimensional linear time-invariant systems. In many practical applications, however, this is not a realistic assumption, and it is essential to take time-delay into consideration in the modelling as the system to be controlled can be highly sensitive to the delay. In this paper, a multi-model switching control algorithm is proposed for retarded time-delay systems. It is assumed that the plant is represented by a family of known multi-input multi-output, controllable and observable linear time-invariant models with multiple delays in the states, and that a bound on the magnitude of the external inputs and disturbances is available. It is shown that under some mild conditions, output tracking can be achieved in the given problem setting.
\end{abstract}

\section{INTRODUCTION}

In conventional adaptive control design, it is typically assumed that the actual plant is fixed, and can be described by a linear time-invariant (LTI) model which is unknown, but that a good deal of a priori information on the plant is known; this information typically includes a knowledge of the upper bound on plant's order, the relative degree, the sign of the high-frequency gain, and minimum phase property. There have been some developments made to relax some of the classical assumptions adopted in conventional adaptive control. For example, some improvements have been made to remove the required information on the sign of the high-frequency gain [1], [2], [3], and to weaken the other assumptions [4], [5]. However, certain assumptions on the right-half plane zeros are required [6].

The adaptive control of systems via switching methods is a relatively new line of research which was motivated to weaken the classical a priori information, and can be traced back to [7], in which a number of questions about the classical assumptions in conventional adaptive control were raised. Switching controllers are nonlinear controllers, which can be used to stabilize and regulate systems with highly uncertain plant models. This is accomplished by using a dictionary of controllers, and by switching from one controller to another at appropriate time instants. There has been a considerable amount of interest towards switching control methods and its applications in the literature recently; e.g., see [8], [9], [10], [11], [12], [13], [14].

In the adaptive switching control approach using a family of plants, it is typically assumed that the plant is not necessarily fixed, i.e. the plant may change from one plant model to another; in this case, it is assumed that the plant model belongs to a known set of models, and so, to implement the adaptive controller, the first step required, is to design (using either a model based, or an experimental approach) a finite set of controllers which provide the required performance for this set of plant models [8], [10], [12], [15], [16], [17]. Then, on applying a so called "switching scheme", each controller is applied to the plant sequentially, and eventually, in finite time, the switching controller stops switching. This implies that as long as the plant remains unchanged, the switching controller will remain locked on one of the appropriate controllers which fulfills the closed-loop performance requirements.

Fu and Barmish [15] considered a compact set of LTI models to represent a plant and imposed an a priori upper bound on the order of plants in this set. They showed that Lyapunov stability can be achieved in this case, by applying a finite set of controllers. Davison and Miller [8] reduced this a priori information, to the knowledge required about the order of a LTI stabilizing compensator. They then simplified the compactness assumption required on the set of possible plant models to just a finite set of plant models. As a result of this, one can design a high-performance LTI controller, e.g. an optimal controller, for each plant model in the known set.

In [12], a class of multi-variable switching control algorithms was introduced which does not require a knowledge of the actual family of plant models. Using this procedure, the only information which is required to be known, is a set of controllers corresponding to the set of plant models, which contains a stabilizing controller for each plant model. A comprehensive survey of switching control systems is presented in [18]. These methods can be very effective when wide-band tracking or disturbance rejection of a physical plant, which can be described by a family of plant models, is required.

All methods described above, assume that the model of the LTI plant being studied is finite dimensional, which is unrealistic in many "real world" applications, such as chemical processes, biological systems, power networks, formation flying, communication networks, to name only a few. One should take the effect of delay into account in control design, as 
the stability margin of the overall system can be highly sensitive to delay. In other words, neglecting the effect of delay in the model of the system can cause degradation of the system performance and may lead to instability. This gives motivation to the present work, which studies the switching control of LTI plants with uncertain delay.

Controller design for fixed model time-delay systems has extensively been investigated in the literature recently [19], [20], [21]. In this paper, it is assumed that the plant is described by a continuous-time retarded time-delay LTI model, which is contained in a specified family of plant models. It is also assumed that a family of controllers has been found to satisfactorily control the models contained in the known set. A switching control scheme is then proposed that uses the input-output information of the candidate controllers. The present work is an extension of the switching control scheme proposed by Miller and Davison in [8] for finite dimensional LTI systems.

This paper is organized as follows. The problem formulation is given in Section II. Then, in Section III, a method is proposed to obtain an upper-bound signal for the error in two phases. This upper-bound signal is essential in finding the switching instants which are later used to develop the switching scheme. An illustrative example is presented in Section IV, which demonstrates the effectiveness of the proposed switching technique. Finally, the concluding remarks are given in Section V.

\section{PROBlem Formulation}

Consider a family of $p$ plants $\mathbf{P}:=\left\{\mathrm{P}_{1}, \ldots, \mathrm{P}_{p}\right\}$. Suppose that each plant $\mathrm{P}_{i}, i \in \overline{\mathrm{p}}:=\{1,2, \ldots, p\}$, in the family is a timedelay, controllable, observable linear time-invariant (LTI) system which is represented by the following retarded differential equation

$$
\begin{aligned}
\dot{x}(t) & =A_{i}^{0} x(t)+\sum_{j=1}^{m} A_{i}^{j} x\left(t-h_{i}^{j}\right)+B_{i} u(t)+E_{i} \omega(t) \\
y(t) & =C_{i} x(t)+F_{i} \omega(t)
\end{aligned}
$$

where $x(t) \in \mathbb{R}^{n_{i}}$ is the state, $u(t) \in \mathbb{R}^{v}$ is the control input, $y(t) \in \mathbb{R}^{r}$ is the output, and $\omega(t) \in \mathbb{R}^{\zeta}$ is the exogenous disturbance. $h_{i}^{j}$,s are the delays in the state of the plant $\mathrm{P}_{i}$, which are assumed to be constant, known and satisfy the inequality $0<h_{i}^{1}<\ldots<h_{i}^{m}:=h_{i}$. It is also assumed that $\omega(t)$ is a bounded piecewise continuous disturbance signal.

Assume now that for each $\mathrm{P}_{i}, i \in \overline{\mathrm{p}}$, a high-performance LTI controller $\mathrm{K}_{i}$ is designed with the following state-space representation (for example, one may use the techniques introduced in [23] and [24])

$$
\begin{aligned}
& \dot{z}(t)=G_{i} z(t)+H_{i} y(t)+J_{i} y_{r e f}(t) \\
& u(t)=K_{i} z(t)+L_{i} y(t)+M_{i} y_{r e f}(t)
\end{aligned}
$$

where $z(t) \in \mathbb{R}^{l}$ is the state of the controller (it is assumed without loss of generality, that all controllers have the same order [8]), and $y_{\text {ref }}$ is a bounded reference signal. Define also the following vectors

$$
\tilde{x}:=\left[\begin{array}{l}
x \\
z
\end{array}\right], \quad \tilde{u}:=\left[\begin{array}{c}
u \\
\dot{z}
\end{array}\right], \quad \tilde{y}:=\left[\begin{array}{c}
y \\
z \\
y_{\text {ref }}
\end{array}\right]
$$

The dynamic feedback control problem corresponding to the pair $\left(\mathrm{P}_{i}, \mathrm{~K}_{i}\right)$ can now be expressed as the the static feedback control problem corresponding to the pair $\left(\tilde{\mathrm{P}}_{i}, \tilde{\mathrm{K}}_{i}\right)$, where $\tilde{\mathrm{P}}_{i}$ and $\tilde{\mathrm{K}}_{i}$ are defined by (3) and (4), respectively

$$
\begin{gathered}
\tilde{u}=\tilde{K}_{i} \tilde{y} \\
\dot{\tilde{x}}(t)=\tilde{A_{i}^{0}} \tilde{x}(t)+\sum_{j=1}^{m} \tilde{A_{i}^{j}} \tilde{x}\left(t-h_{i}^{j}\right)+\tilde{B}_{i} \tilde{u}(t)+\tilde{E}_{i} \omega(t) \\
\tilde{y}(t)=\tilde{C}_{i} \tilde{x}(t)+\tilde{D}_{i} y_{r e f}(t)+\tilde{F}_{i} \omega(t)
\end{gathered}
$$

where

$$
\begin{gathered}
\tilde{A}_{i}^{0}=\left[\begin{array}{cc}
A_{i}^{0} & 0 \\
0 & 0
\end{array}\right], \quad \tilde{A}_{i}^{j}=\left[\begin{array}{cc}
A_{i}^{j} & 0 \\
0 & 0
\end{array}\right], \quad \tilde{B}_{i}=\left[\begin{array}{cc}
B_{i} & 0 \\
0 & I
\end{array}\right] \\
\tilde{C}_{i}=\left[\begin{array}{cc}
C_{i} & 0 \\
0 & I \\
0 & 0
\end{array}\right], \quad \tilde{D}_{i}=\left[\begin{array}{l}
0 \\
0 \\
I
\end{array}\right], \quad \tilde{F}_{i}=\left[\begin{array}{c}
F_{i} \\
0 \\
0
\end{array}\right] \\
\tilde{E}_{i}=\left[\begin{array}{c}
E_{i} \\
0
\end{array}\right], \quad \tilde{K}_{i}=\left[\begin{array}{ccc}
L_{i} & K_{i} & M_{i} \\
H_{i} & G_{i} & J_{i}
\end{array}\right]
\end{gathered}
$$


It is to be noted that all of the roots of the following characteristic equation

$$
\tilde{\Delta}_{i}(s)=\operatorname{det}\left(s I-\tilde{A}_{i}^{0}-\tilde{B}_{i} \tilde{K}_{i} \tilde{C}_{i}-\sum_{j=1}^{m} \tilde{A}_{i}^{j} e^{-h_{i}^{j} s}\right)=0
$$

lie in the open left-half of the complex plane. The objective of this paper is to propose a switching mechanism so that output tracking is achieved in the presence of external disturbances. In other words, it is desired to switch between the feedback gains $\tilde{\mathrm{K}}_{i}$ at appropriate time instants so that the output error approaches zero as $t \rightarrow \infty$.

Remark 1: Throughout this paper and prior to Corollary 2, the only requirement for designing high-performance controllers is that each $\mathrm{K}_{i}$ stabilizes the corresponding plant $\mathrm{P}_{i}$. For the stability of time-delay systems, see [22]-[24]. To have exact tracking results (Corollary 2), more requirements on $\mathrm{K}_{i}$ are expected (Assumption 3).

\section{PReliminaries}

It is known from the functional differential equations theory [25] that the solution of the retarded state equation

$$
\begin{aligned}
& \dot{x}(t)=A_{i}^{0} x(t)+\sum_{j=1}^{m} A_{i}^{j} x\left(t-h_{i}^{j}\right)+f(t) \\
& \phi(r)=x(r), \quad-h_{i} \leq r \leq 0
\end{aligned}
$$

in terms of $f(t)$ and initial function $\phi(r)$ can be written as

$$
x(t)=x(\phi, 0)+\int_{0}^{t} X_{i}(t-\tau) f(\tau) d \tau
$$

where the homogenous part of the solution in (7), i.e. $x(\phi, 0)$, is given by

$$
x(\phi, 0)=X_{i}(t) \phi(0)+\sum_{j=1}^{m} \int_{-h_{i}^{j}}^{0} X_{i}\left(t-r-h_{i}^{j}\right) A_{i}^{j} \phi(r) d r
$$

$X_{i}(t)$ in (7) and (8) is the fundamental matrix for the corresponding retarded state equation which satisfies the following matrix differential equation [26]

$$
\dot{X}_{i}(t)=A_{i}^{0} X_{i}(t)+\sum_{j=1}^{m} A_{i}^{j} X_{i}\left(t-h_{i}^{j}\right)
$$

with the initial condition given by

$$
X_{i}(r)=\left\{\begin{array}{ll}
I_{q_{i}}, & r=0 \\
0_{q_{i}}, & r \in\left[-h_{i} \leq r<0\right)
\end{array}, \quad q_{i}:=n_{i}+l, \quad i \in \overline{\mathrm{p}}\right.
$$

where $I_{q_{i}}$ denotes the $q_{i} \times q_{i}$ identity matrix, and $0_{q_{i}}$ is the $q_{i} \times q_{i}$ zero matrix. Furthermore, it is known that there exist constants $\alpha_{i}$ and $\lambda_{i}$, so that [25]

$$
\left\|X_{i}(t)\right\| \leq \alpha_{i} e^{\lambda_{i} t}, \quad \forall t>0, \quad i \in \overline{\mathrm{p}}
$$

where $\|$.$\| represents the 2-norm of a vector, or the corresponding induced 2-norm of a matrix. As a result, it can be easily$ concluded that there exists a constant $\sigma_{i}$, such that

$$
\|x(\phi, 0)\| \leq \sigma_{i} e^{\lambda_{i} t} \times \max _{-h_{i} \leq r \leq 0}\|\phi(r)\|, \quad \forall t>0, \quad i \in \overline{\mathrm{p}}
$$

Moreover, consider the characteristic equation corresponding to the retarded state equation (6a) as follows

$$
\Delta_{i}(s)=\operatorname{det}\left(s I-\tilde{A}_{i}^{0}-\sum_{j=1}^{m} \tilde{A}_{i}^{j} e^{-h_{i}^{j} s}\right)=0
$$

and define $\lambda_{0_{i}}$ as

$$
\lambda_{0_{i}}=\max \left\{\operatorname{Re}\{s\}: \Delta_{i}(s)=0\right\}
$$

Then, it can be easily verified that $\lambda_{i}$ in (9) is greater than or equal to $\lambda_{0_{i}}$ [25]. Consequently, If the system given by (6a) is asymptotically stable, then one can choose $\lambda_{i}$ in (9) as a strictly negative value.

\section{Main Results}

In this section, the switching control scheme given in [8] is modified to account for the delay in the state of the system. 


\section{A. Finding an Upper Bound on the Initial Function}

Lemma 1: Suppose that the system (1) is observable. Let the initial function be denoted by $\phi(r)$, where $r \in\left[-h_{i}, 0\right]$. Then for every arbitrary $T>0$ and $i \in \overline{\mathrm{p}}$, the matrix $Q_{i}(r, T)$ defined by

$$
Q_{i}(r, T):=\int_{0}^{T} \int_{-h_{i}}^{0^{+}} \Theta_{i}^{\prime}(t, \tau) C_{i}^{\prime} C_{i} \Theta_{i}(t, r) d \tau d t
$$

is invertible, where

$$
\Theta_{i}(t, r):=X_{i}(t-r) \delta(r)+\sum_{j=1}^{m} X_{i}\left(t-r-h_{i}^{j}\right) A_{i}^{j} u_{-1}\left(r-h_{i}^{j}\right)
$$

and $X_{i}(t)$ is the fundamental matrix for the corresponding retarded differential equation of plant $\mathrm{P}_{i}\left(\delta(\right.$.$) and u_{-1}($.$) are$ Dirac delta and unit step functions, respectively).

Proof: If $u(t)$ and $\omega(t)$ are identically zero in the interval $[0, T]$, the output of the system (1) can be obtained as follows

$$
y(t)=C_{i}\left(X_{i}(t) \phi(0)+\sum_{j=1}^{m} \int_{-h_{i}^{j}}^{0} X_{i}\left(t-r-h_{i}^{j}\right) A_{i}^{j} \phi(r) d r\right)
$$

Using (13) and the sifting property of Dirac delta, $y(t)$ can be rewritten as

$$
y(t)=C_{i} \int_{-h_{i}}^{0^{+}} \Theta_{i}(t, r) \phi(r) d r
$$

Multiplying both sides of (15) by $\Theta_{i}{ }^{\prime}(t, \tau) C_{i}{ }^{\prime}$ and integrating over $t$ and $\tau$ result in

$$
\begin{aligned}
\int_{-h_{i}}^{0^{+}} \int_{0}^{T} \Theta_{i}{ }^{\prime}(t, \tau) C_{i}{ }^{\prime} y(t) d t d \tau & =\int_{-h_{i}}^{0^{+}} \int_{0}^{T} \Theta_{i}^{\prime}(t, \tau) C_{i}{ }^{\prime} C_{i} \int_{-h_{i}}^{0^{+}} \Theta_{i}(t, r) \phi(r) d r d t d \tau \\
& =\int_{-h_{i}}^{0^{+}}\left[\int_{0}^{T} \int_{-h_{i}}^{0^{+}} \Theta_{i}{ }^{\prime}(t, \tau) C_{i}{ }^{\prime} C_{i} \Theta_{i}(t, r) d \tau d t\right] \phi(r) d r
\end{aligned}
$$

From the definition given by (12), the following can be obtained

$$
\int_{-h_{i}}^{0^{+}} \int_{0}^{T} \Theta_{i}^{\prime}(t, r) C_{i}^{\prime} y(t) d t d r=\int_{-h_{i}}^{0^{+}} Q_{i}(r, T) \phi(r) d r
$$

Suppose now, that $Q_{i}(r, T)$ is not full-rank for some $r_{0} \in\left[-h_{i}, 0\right]$. Then a nonzero vector $\varphi_{0}$ exists, such that $Q_{i}\left(r_{0}, T\right) \varphi_{0}=0$. Therefore, if $y(t)$ is identically zero for all $t \in[0, T]$, then $\phi(r)=\varphi_{0} \delta\left(r-r_{0}\right)$ and $\phi(r)=0$ will be two different solutions for (16). On the other hand, since the system (1) is observable, the equation (16) will have a unique solution for $\phi(r)$. This means that the observability assumption is violated and thus, it can be concluded that $Q_{i}(r, T)$ should be invertible for all $r \in\left[-h_{i}, 0\right]$.

Corollary 1: Suppose that the system (1) is observable. If $u(t)$ and $\omega(t)$ are identically zero in the interval $[0, T]$, it follows that the vector $\phi(r)$ given by

$$
\phi(r)=Q_{i}^{-1}(r, T) \int_{0}^{T} \Theta_{i}^{\prime}(t, r) C_{i}^{\prime} y(t) d t
$$

is the unique solution of (16).

Proof: It follows from Lemma 1 that the inverse of $Q_{i}(r, T)$ exists and thus, (17) gives a solution for $\phi(r)$ in (16). In addition, from the observability assumption, it can be concluded that this solution must be unique.

It is to be noted that Lemma 1 provides only a sufficient condition for non-singularity of the matrix (12). The matrix $Q_{i}(r, T)$ is known as the observability gramian for the time-delay system (1). One can use the methods given in [27] and [28] to check the observability of time-delay systems.

Lemma 2: Consider the observable system (1), and assume that $u(t)=0$ for all $t \in[0, T]$, where $T$ is any arbitrary positive nonzero value. Then, there exists a constant $\beta_{i}$, so that for any arbitrary continuous initial condition $\phi(r)$ and every disturbance $\omega(t)$

$$
\max _{-h_{i} \leq r \leq 0}\|\phi(r)\| \leq \beta_{i} \sup _{t \geq 0}\|\omega(t)\|+\sup _{-h_{i} \leq r<0} \frac{1}{\eta_{i}(r)}\left\|\int_{0}^{T} \Theta_{i}^{\prime}(t, r) C_{i}^{\prime} y(t) d t\right\|
$$

where $\eta_{i}(r)$ is the smallest singular value of $Q_{i}(r, T)$, and

$$
\begin{aligned}
\beta_{i} & =\sup _{-\bar{h}_{i} \leq r<0} \frac{1}{\eta_{i}(r)} \int_{0}^{T}\left\|\left(\int_{t}^{T} \Theta_{i}{ }^{\prime}(\tau, r) C_{i}{ }^{\prime} C_{i} X_{i}(\tau-t) E_{i} d \tau\right)+\Theta_{i}{ }^{\prime}(t, r) C_{i}{ }^{\prime} F_{i}\right\| d t \\
\Upsilon_{i}(y) & =\left\|\int_{0}^{T} \Theta_{i}{ }^{\prime}(t, r) C_{i}{ }^{\prime} y(t) d t\right\|
\end{aligned}
$$


Proof: Using an approach similar to the proof of Lemma 1, it can be shown that

$$
\int_{-h_{i}}^{0^{+}} \int_{0}^{T} \Theta_{i}{ }^{\prime}(t, r) C_{i}{ }^{\prime}\left(y(t)-F_{i} \omega(t)\right) d t d r=\int_{-h_{i}}^{0^{+}} Q_{i}(r, T) \phi(r) d r+\int_{-h_{i}}^{0^{+}} \int_{0}^{T} \int_{0}^{t} \Theta_{i}{ }^{\prime}(t, r) C_{i}{ }^{\prime} C_{i} X_{i}(t-\tau) E_{i} \omega(\tau) d \tau d t d r
$$

Since the system (1) is observable, it follows from Lemma 1 that

where

$$
\phi(r)=Q_{i}^{-1}(r, T)\left[\int_{0}^{T} \Theta_{i}{ }^{\prime}(t, r) C_{i}^{\prime} y(t) d t-\int_{0}^{T} \Theta_{i}{ }^{\prime}(t, r) C_{i}^{\prime} F_{i} \omega(t) d t-\xi(r)\right]
$$

(2)

$$
\left.\xi(r)=\int_{0}^{T} \int_{0}^{t} \Theta_{i}^{\prime}(t, r) C_{i}^{\prime} C_{i} X_{i}(t-\tau) E_{i} \omega(\tau) d \tau d t\right]
$$

or equivalently,

$$
\begin{aligned}
\xi(r) & =\int_{0}^{T} \int_{\tau}^{T} \Theta_{i}{ }^{\prime}(t, r) C_{i}{ }^{\prime} C_{i} X_{i}(t-\tau) E_{i} \omega(\tau) d t d \tau \\
& =\int_{0}^{T}\left[\int_{\tau}^{T} \Theta_{i}{ }^{\prime}(t, r) C_{i}{ }^{\prime} C_{i} X_{i}(t-\tau) E_{i} d t\right] \omega(\tau) d \tau \\
& =\int_{0}^{T}\left[\int_{t}^{T} \Theta_{i}{ }^{\prime}(\tau, r) C_{i}{ }^{\prime} C_{i} X_{i}(\tau-t) E_{i} d \tau\right] \omega(t) d t
\end{aligned}
$$

Consequently, $\phi(r)$ can be obtained as follows

$$
\begin{aligned}
\phi(r)= & \int_{0}^{T} Q_{i}{ }^{-1}(r, T) \Theta_{i}{ }^{\prime}(t, r) C_{i}{ }^{\prime} y(t) d t \\
& -\int_{0}^{T} Q_{i}{ }^{-1}(r, T)\left[\Theta_{i}{ }^{\prime}(t, r) C_{i}{ }^{\prime} F_{i}+\int_{t}^{T} \Theta_{i}{ }^{\prime}(\tau, r) C_{i}{ }^{\prime} C_{i} X_{i}(\tau-t) E_{i} d \tau\right] \omega(t) d t
\end{aligned}
$$

where $r \in\left[-h_{i}, 0\right]$. By taking the norm of both sides of the above equation, using the related inequalities, and noting that $\left\|Q_{i}^{-1}(r, T)\right\|=1 / \eta_{i}(r)$, the upper bound for $\|\phi(r)\|$ given in Lemma 2 is obtained. Note that since the function $\phi(r)$ is assumed to be continuous

$$
\max _{-h_{i} \leq r \leq 0} \phi(r) \leq \sup _{-h_{i} \leq r<0} \phi(r)
$$

Remark 2: To find the upper bound function given in Lemma 2, it is not required to obtain the inverse of the observability gramian matrix $Q_{i}(r, T)$. This reduces the computational complexity of the proposed switching algorithm. Nevertheless, integration of matrix exponentials is numerically difficult, in general. It is shown in the following two propositions that the upper bounds on $\beta_{i}$ and $\Upsilon_{i}(y)$ in (18) can be found directly without matrix integration.

Proposition 1: The constant $\beta_{i}$ defined in Lemma 2 satisfies the following inequality

$$
\begin{aligned}
\beta_{i} \leq & \sup _{-h_{i} \leq r<0} \frac{1}{\eta_{i}(r)}\left[\int_{0}^{T} \int_{t}^{T} \sum_{j=1}^{m} u_{-1}\left(r-h_{i}^{j}\right)\left\|A_{i}^{j^{\prime}}\right\| \alpha_{i} e^{\lambda_{i}\left(\tau-r-h_{i}^{j}\right)} u_{-1}\left(\tau-r-h_{i}^{j}\right)\left\|C_{i}^{\prime}\right\|\left\|C_{i}\right\| \alpha_{i} e^{\lambda_{i}(\tau-t)}\left\|E_{i}\right\| d \tau d t\right. \\
& \left.+\int_{0}^{T} \sum_{j=1}^{m} u_{-1}\left(r-h_{i}^{j}\right)\left\|A_{i}^{j^{\prime}}\right\| \alpha_{i} e^{\lambda_{i}\left(t-r-h_{i}^{j}\right)} u_{-1}\left(t-r-h_{i}^{j}\right)\left\|C_{i}^{\prime}\right\|\left\|F_{i}\right\| d t\right]
\end{aligned}
$$

Proof: Applying triangle inequality to (18a) yields

$$
\beta_{i} \leq \sup _{-h_{i} \leq r<0} \frac{1}{\eta_{i}(r)}\left[\int_{0}^{T}\left\|\int_{t}^{T} \Theta_{i}^{\prime}(\tau, r) C_{i}^{\prime} C_{i} X_{i}(\tau-t) E_{i} d \tau\right\| d t+\int_{0}^{T}\left\|\Theta_{i}^{\prime}(t, r) C_{i}^{\prime} F_{i}\right\| d t\right]
$$

Thus

$$
\beta_{i} \leq \sup _{-h_{i} \leq r<0} \frac{1}{\eta_{i}(r)}\left[\int_{0}^{T} \int_{t}^{T}\left\|\Theta_{i}{ }^{\prime}(\tau, r)\right\|\left\|C_{i}{ }^{\prime}\right\|\left\|C_{i}\right\|\left\|X_{i}(\tau-t)\right\|\left\|E_{i}\right\| d \tau d t+\int_{0}^{T}\left\|\Theta_{i}^{\prime}(t, r)\right\|\left\|C_{i}{ }^{\prime}\right\|\left\|F_{i}\right\| d t\right]
$$

It follows from (13) that

$$
\begin{aligned}
\beta_{i} \leq & \sup _{-h_{i} \leq r<0} \frac{1}{\eta_{i}(r)}\left[\int_{0}^{T} \int_{t}^{T} \sum_{j=1}^{m} u_{-1}\left(r-h_{i}^{j}\right)\left\|A_{i}^{j^{\prime}}\right\|\left\|X_{i}^{\prime}\left(\tau-r-h_{i}^{j}\right)\right\|\left\|C_{i}^{\prime}\right\|\left\|C_{i}\right\|\left\|X_{i}(\tau-t)\right\|\left\|E_{i}\right\| d \tau d t\right. \\
& \left.+\int_{0}^{T} \sum_{j=1}^{m} u_{-1}\left(r-h_{i}^{j}\right)\left\|A_{i}^{j^{\prime}}\right\|\left\|X_{i}^{\prime}\left(t-r-h_{i}^{j}\right)\right\|\left\|C_{i}^{\prime}\right\|\left\|F_{i}\right\| d t\right]
\end{aligned}
$$


The following inequality is then resulted from (9)

$$
\begin{aligned}
\beta_{i} \leq & \sup _{-h_{i} \leq r<0} \frac{1}{\eta_{i}(r)}\left[\int_{0}^{T} \int_{t}^{T} \sum_{j=1}^{m} u_{-1}\left(r-h_{i}^{j}\right)\left\|A_{i}^{j^{\prime}}\right\| \alpha_{i} e^{\lambda_{i}\left(\tau-r-h_{i}^{j}\right)} u_{-1}\left(\tau-r-h_{i}^{j}\right)\left\|C_{i}^{\prime}\right\|\left\|C_{i}\right\| \alpha_{i} e^{\lambda_{i}(\tau-t)}\left\|E_{i}\right\| d \tau d t\right. \\
& \left.+\int_{0}^{T} \sum_{j=1}^{m} u_{-1}\left(r-h_{i}^{j}\right)\left\|A_{i}^{j^{\prime}}\right\| \alpha_{i} e^{\lambda_{i}\left(t-r-h_{i}^{j}\right)} u_{-1}\left(t-r-h_{i}^{j}\right)\left\|C_{i}^{\prime}\right\|\left\|F_{i}\right\| d t\right]
\end{aligned}
$$

Proposition 2: $\Upsilon_{i}(y)$ defined in Lemma 2 satisfies the following inequality

$$
\Upsilon_{i}(y) \leq \int_{0}^{T} \sum_{j=1}^{m} u_{-1}\left(r-h_{i}^{j}\right)\left\|A_{i}^{j^{\prime}}\right\| \alpha_{i} e^{\lambda_{i}\left(t-r-h_{i}^{j}\right)} u_{-1}\left(t-r-h_{i}^{j}\right) \times\left\|C_{i}^{\prime}\right\|\|y(t)\| d t
$$

Proof: Applying triangle inequality to (18b) yields

$$
\Upsilon_{i}(y) \leq \int_{0}^{T}\left\|\Theta_{i}^{\prime}(t, r)\right\|\left\|C_{i}^{\prime}\right\|\|y(t)\| d t
$$

It follows from (13) that

$$
\Upsilon_{i}(y) \leq \int_{0}^{T} \sum_{j=1}^{m} u_{-1}\left(r-h_{i}^{j}\right)\left\|A_{i}^{j^{\prime}}\right\|\left\|X_{i}^{\prime}\left(t-r-h_{i}^{j}\right)\right\|\left\|C_{i}^{\prime}\right\|\|y(t)\| d t
$$

The following inequality can then be obtained from (9),

$$
\Upsilon_{i}(y) \leq \int_{0}^{T} \sum_{j=1}^{m} u_{-1}\left(r-h_{i}^{j}\right)\left\|A_{i}^{j^{\prime}}\right\| \alpha_{i} e^{\lambda_{i}\left(t-r-h_{i}^{j}\right)} u_{-1}\left(t-r-h_{i}^{j}\right)\left\|C_{i}^{\prime}\right\|\|y(t)\| d t
$$

It is shown in the next proposition that the proper choice of $T$ can be very important in computing of $Q_{i}(r, T)$ more efficiently.

Proposition 3: If the time interval $T$ is chosen smaller than the smallest delay in the states of each of the models, i.e. $T<\min \left\{h_{1}^{1}, h_{2}^{1}, \ldots, h_{p}^{1}\right\}$, then the matrix $Q_{i}(r, T),-h_{i} \leq r<0$, can be obtained as follows

$$
Q_{i}(r, T)=\psi_{1}{ }^{i}(r, T)+\psi_{2}{ }^{i}(r, T)
$$

where

$$
\begin{aligned}
& \psi_{1}{ }^{i}(r, T)=\sum_{l=1}^{m} u_{-1}\left(r+h_{i}^{l}\right) u_{-1}\left(T-h_{i}^{l}-r\right) \times\left[\int_{r+h_{i}^{l}}^{T} e^{A_{i}^{0^{\prime}} t} C_{i}^{\prime} C_{i} e^{A_{i}^{0} t} d t\right] e^{-A_{i}^{0} r} e^{-A_{i}^{0} h_{i}^{l}} A_{i}^{l} \\
& \psi_{2}{ }^{i}(r, T)=\left[A_{i}^{0^{\prime}}\right]^{-1} \sum_{j=1}^{m} \sum_{l=1}^{m} u_{-1}\left(r+h_{i}^{l}\right) u_{-1}\left(T-h_{i}^{l}-r\right) A_{i}^{j^{\prime}} \times\left[\int_{r+h_{i}^{l}}^{T} e^{A_{i}^{0^{\prime}}{ }^{\prime} t} C_{i}^{\prime} C_{i} e^{A_{i}^{0} t} d t-\int_{r+h_{i}^{l}}^{T} C_{i}^{\prime} C_{i} e^{A_{i}^{0} t} d t\right] e^{-A_{i}^{0}\left(r+h_{i}^{l}\right)} A_{i}^{l}
\end{aligned}
$$

Proof: By substituting (13) into (12), the matrix $Q_{i}(r, T)$, for $-h \leq r<0$, can be rewritten as

$$
\begin{aligned}
Q_{i}(r, T):= & \sum_{l=1}^{m} \int_{0}^{T} X_{i}^{\prime}(t) C_{i}^{\prime} C_{i} X_{i}\left(t-r-h_{i}^{l}\right) A_{i}^{l} u_{-1}\left(r+h_{i}^{l}\right) d t \\
& +\sum_{j=1}^{m} \sum_{l=1}^{m} \int_{0}^{T} \int_{-\bar{h}_{i}}^{0} u_{-1}\left(\tau+h_{i}^{j}\right) A_{i}^{j^{\prime}} X_{i}^{\prime}\left(t-\tau-h_{i}^{j}\right) C_{i}^{\prime} C_{i} X_{i}\left(t-r-h_{i}^{l}\right) A_{i}^{l} u_{-1}\left(r+h_{i}^{l}\right) d \tau d t
\end{aligned}
$$

If $\bar{\psi}_{1}^{i}(r)$ and $\bar{\psi}_{2}^{i}(r)$ are defined as below

$$
\begin{aligned}
\bar{\psi}_{1}^{i}(r) & :=\sum_{l=1}^{m} \int_{0}^{T} X_{i}^{\prime}(t) C_{i}^{\prime} C_{i} X_{i}\left(t-r-h_{i}^{l}\right) A_{i}^{l} u_{-1}\left(r+h_{i}^{l}\right) d t \\
\bar{\psi}_{2}^{i}(r) & :=\sum_{j=1}^{m} \sum_{l=1}^{m} \int_{0}^{T} \int_{-\bar{h}_{i}}^{0} u_{-1}\left(\tau+h_{i}^{j}\right) A_{i}^{j^{\prime}} X_{i}^{\prime}\left(t-\tau-h_{i}^{j}\right) C_{i}^{\prime} C_{i} X_{i}\left(t-r-h_{i}^{l}\right) A_{i}^{l} u_{-1}\left(r+h_{i}^{l}\right) d \tau d t
\end{aligned}
$$

$Q_{i}(r, T)$ can be written as $\bar{\psi}_{1}^{i}(r)+\bar{\psi}_{2}^{i}(r)$. On the other hand, it is known that for $t<h_{i}^{1}$,

$$
X_{i}(t)=e^{A_{i}^{0} t} u_{-1}(t)
$$

Substituting $X_{i}(t)$ given by (22) into (21), one can easily verify that $\bar{\psi}_{1}^{i}(r)=\psi_{1}^{i}(r)$. In addition, $\bar{\psi}_{2}^{i}(r)$ can be simplified as

$$
\bar{\psi}_{2}^{i}(r)=\sum_{j=1}^{m} \sum_{l=1}^{m} A_{i}^{j^{\prime}} \int_{0}^{T}\left(\int_{-h_{i}^{j}}^{t-h_{i}^{j}} e^{-A_{i}^{0^{\prime}}\left(\tau+h_{i}^{j}\right)} d \tau\right) e^{A_{i}^{0^{\prime} t}} C_{i}^{\prime} C_{i} e^{A_{i}^{0}\left(t-r-h_{i}^{l}\right)} A_{i}^{l} u_{-1}\left(r+h_{i}^{l}\right) d t
$$


or equivalently,

$$
\bar{\psi}_{2}^{i}(r)=\sum_{j=1}^{m} \sum_{l=1}^{m} A_{i}^{j^{\prime}} \int_{0}^{T}\left(\int_{-h_{i}^{j}}^{t-h_{i}^{j}} e^{-A_{i}^{0^{\prime}}\left(\tau+h_{i}^{j}\right)} d \tau\right) e^{A_{i}^{0^{\prime}}{ }^{t}} C_{i}^{\prime} C_{i} e^{A_{i}^{0}\left(t-r-h_{i}^{l}\right)} u_{-1}\left(t-r-h_{i}^{l}\right) A_{i}^{l} u_{-1}\left(r+h_{i}^{l}\right) d t
$$

It follows then by integrating with respect to $\tau$ that

$$
\bar{\psi}_{2}^{i}(r)=\sum_{j=1}^{m} \sum_{l=1}^{m} A_{i}^{j^{\prime}} \int_{0}^{T}\left[A_{i}^{0^{\prime}}\right]^{-1}\left(e^{A_{i}^{0^{\prime}} t}-I\right) C_{i}^{\prime} C_{i} e^{A_{i}^{0}\left(t-r-h_{i}^{l}\right)} u_{-1}\left(t-r-h_{i}^{l}\right) A_{i}^{l} u_{-1}\left(r+h_{i}^{l}\right) d t
$$

It can be concluded that

$$
\bar{\psi}_{2}^{i}(r)=\left[A_{i}^{0^{\prime}}\right]^{-1} \sum_{j=1}^{m} \sum_{l=1}^{m} u_{-1}\left(r+h_{i}^{l}\right) A_{i}^{j^{\prime}}\left[\int_{r+h_{i}^{l}}^{T} e^{A_{i}^{0^{\prime} t}} C_{i}^{\prime} C_{i} e^{A_{i}^{0} t} u_{-1}\left(t-r-h_{i}^{l}\right) d t-\int_{r+h_{i}^{l}}^{T} C_{i}^{\prime} C_{i} e^{A_{i}^{0} t} u_{-1}\left(t-r-h_{i}^{l}\right) d t\right] e^{-A_{i}^{0}\left(r+h_{i}^{l}\right)} A_{i}^{l}
$$

Therefore, $\bar{\psi}_{2}^{i}(r)=\psi_{2}{ }^{i}(r)$. This completes the proof.

Remark 3: The expression obtained for $Q_{i}(r, T)$ in Proposition 3 involves the standard matrix exponential integrals, for which a computationally efficient method is introduced in [29]. This substantially reduces the computational requirements for finding the matrix $Q_{i}(r, T)$.

\section{B. Finding an Upper Bound for the State}

Lemma 3: There exist constants $\gamma_{i_{1}}, \gamma_{i_{2}}, \gamma_{i_{3}}$, and $\lambda_{i}<0$, so that the solution of (4) satisfies

$$
\|\tilde{x}(t)\| \leq \gamma_{i_{1}} e^{\lambda_{i} t} \max _{-h \leq r \leq 0}\|\tilde{\phi}(r)\|+\int_{0}^{t} e^{\lambda_{i}(t-\tau)} \gamma_{i_{3}}\|\tilde{\omega}(\tau)\| d \tau+\int_{0}^{t} e^{\lambda_{i}(t-\tau)}\left[\gamma_{i_{2}}\left\|\tilde{u}(\tau)-\tilde{K}_{i}\left(\tilde{y}(\tau)-\tilde{D}_{i} y_{r e f}(\tau)\right)\right\|\right] d \tau
$$

Proof: The retarded differential equation for $\tilde{x}(t)$ given by (4) can be rewritten as

$$
\dot{\tilde{x}}(t)=\left(\tilde{A}_{i}+\tilde{B}_{i} \tilde{K}_{i} \tilde{C}_{i}\right) \tilde{x}(t)+\left(\tilde{E}_{i}+\tilde{B}_{i} \tilde{K}_{i} \tilde{F}_{i}\right) \tilde{\omega}(t)+\tilde{B}_{i}\left[\tilde{u}(t)-\tilde{K}_{i}\left(\tilde{y}(t)-\tilde{D}_{i} y_{r e f}(t)\right)\right]+\sum_{j=1}^{m} \tilde{A}_{i}^{j} \tilde{x}\left(t-h_{i}^{j}\right)
$$

One can express $\tilde{x}(t)$ using an equation similar to (7), as follows

$$
\tilde{x}(t)=\tilde{x}(\tilde{\phi}, 0)+\int_{0}^{t} \tilde{X}_{i}(t-\tau)\left\{\left(\tilde{E}_{i}+\tilde{B}_{i} \tilde{K}_{i} \tilde{F}_{i}\right) \tilde{\omega}(\tau)+\tilde{B}_{i}\left[\tilde{u}(\tau)-\tilde{K}_{i}\left(\tilde{y}(\tau)-\tilde{D}_{i} y_{r e f}(\tau)\right)\right]\right\} d \tau
$$

where $\tilde{X}_{i}(t), i \in \overline{\mathrm{p}}$ is the fundamental matrix for the retarded state equation in (23). Consequently, it can be concluded that $\|\tilde{x}(t)\|$ satisfies the following inequality

$$
\|\tilde{x}(t)\| \leq\|\tilde{x}(\tilde{\phi}, 0)\|+\int_{0}^{t}\left\|\tilde{X}_{i}(t-\tau)\right\|\left\|\tilde{E}_{i}+\tilde{B}_{i} \tilde{K}_{i} \tilde{F}_{i}\right\|\|\tilde{\omega}(\tau)\| d \tau+\int_{0}^{t}\left\|\tilde{X}_{i}(t-\tau)\right\|\left\|\tilde{B}_{i}\right\|\left\|\tilde{u}(\tau)-\tilde{K}_{i}\left(\tilde{y}(\tau)-\tilde{D}_{i} y_{r e f}(\tau)\right)\right\| d \tau
$$

It is known that there exist constants $\alpha_{i}, \lambda_{i}$, and $\sigma_{i}$ such that the following inequalities hold

$$
\begin{aligned}
\left\|\tilde{X}_{i}(t)\right\| & \leq \alpha_{i} e^{\lambda_{i} t} \\
\|\tilde{x}(\phi, 0)\| & \leq \sigma_{i} e^{\lambda_{i} t} \times \max _{-h_{i} \leq r \leq 0}\|\tilde{\phi}(r)\|
\end{aligned}
$$

Since the closed-loop system in (23) is asymptotically stable (because it corresponds to the pair $\left(\tilde{\mathrm{P}}_{i}, \tilde{\mathrm{K}}_{i}\right)$ ), all of its poles given by the roots of the characteristics equation of (5) lie in open right-half plane. Therefore, $\lambda_{i}$ can be chosen strictly negative. The upper bound for $\|\tilde{x}(t)\|$ is then obtained from (24) and (25), as follows

$$
\begin{aligned}
\|\tilde{x}(t)\| \leq & \sigma_{i} e^{\lambda_{i} t} \max _{-h_{i} \leq r \leq 0}\|\tilde{\phi}(r)\|+\int_{0}^{t} \alpha_{i} e^{\lambda_{i}(t-\tau)}\left\|\tilde{E}_{i}+\tilde{B}_{i} \tilde{K}_{i} \tilde{F}_{i}\right\|\|\tilde{\omega}(\tau)\| d \tau \\
& +\int_{0}^{t} \alpha_{i} e^{\lambda_{i}(t-\tau)}\left\|\tilde{B}_{i}\right\|\left\|\tilde{u}(\tau)-\tilde{K}_{i}\left(\tilde{y}(\tau)-\tilde{D}_{i} y_{r e f}(\tau)\right)\right\| d \tau
\end{aligned}
$$

The proof follows by choosing

$$
\begin{aligned}
& \gamma_{i_{1}}=\sigma_{i} \\
& \gamma_{i_{2}}=\alpha_{i}\left\|\tilde{B}_{i}\right\| \\
& \gamma_{i_{3}}=\alpha_{i}\left\|\tilde{E}_{i}+\tilde{B}_{i} \tilde{K}_{i} \tilde{F}_{i}\right\|
\end{aligned}
$$

Lemma 4: Assume that $\sigma_{i}$ and $\lambda_{i}$ satisfy (25) and let $\alpha_{i}$ be equal to $\sqrt{q_{i}} \sigma_{i}$. Then the following inequality holds

$$
\left\|\tilde{X}_{i}(t)\right\| \leq \alpha_{i} e^{\lambda_{i} t}
$$


Proof: It is known that

$$
\left\|\tilde{X}_{i}(t)\right\| \leq\left\|\tilde{X}_{i}(t)\right\|_{F}
$$

where $\|\cdot\|_{F}$ denotes the Frobenius norm. On the other hand,

$$
\left\|\tilde{X}_{i}(t)\right\|_{F}^{2}=\sum_{s=1}^{q_{i}}\left\|\tilde{x}\left(\tilde{\phi}_{i}^{s}, 0\right)\right\|^{2}
$$

where $\tilde{\phi}_{i}^{s}, s=1,2, \ldots, q_{i}$ and $i \in \overline{\mathrm{p}}$, is defined as follows

$$
\tilde{\phi}_{i}^{s}(r)= \begin{cases}e_{s}, & r=0 \\ 0_{q_{i}}, & r \in\left[-h_{i}, 0\right)\end{cases}
$$

The vector $e_{s}$ in the above definition is the $s^{\text {th }}$ column of the $q_{i} \times q_{i}$ identity matrix. In addition, from (25)

$$
\left\|\tilde{x}\left(\tilde{\phi}_{s}, 0\right)\right\|^{2} \leq \sigma_{i}^{2} e^{2 \lambda_{i} t}
$$

for all $s=1, \ldots, q_{i}$. The following inequality is directly obtained from (27) and (28)

$$
\left\|\tilde{X}_{i}(t)\right\|_{F}^{2} \leq q_{i} \sigma_{i}^{2} e^{2 \lambda_{i} t}
$$

The proof follows immediately from (26) and (29).

The following procedure can be used to obtain the constants $\lambda_{i}, \sigma_{i}$, and $\alpha_{i}$ such that the inequalities (24) and (25) in Lemma 3 hold.

1) Use the Mikhailov diagram [30] to find the smallest negative value for $\lambda_{i}$.

2) Find the constant $\sigma_{i}$, based on the value obtained for $\lambda_{i}$ in step 1 and by using the following relations [30]:

$$
\begin{aligned}
& \sigma_{i}=\sqrt{\frac{\alpha_{i_{2}}}{\alpha_{i_{1}}}}, \quad \alpha_{i_{1}}=\lambda_{\min }\left(R_{i}\right), \\
& \alpha_{i_{2}}=\lambda_{\max }\left(R_{i}\right)+\sum_{j=1}^{m} h_{i}^{j} \lambda_{\max }\left(S_{i}^{j}\right)
\end{aligned}
$$

where $R_{i}$ and $Q_{i}^{j}, j=1, \ldots, m$, which are real $q_{i} \times q_{i}$ positive-definite matrices, satisfy the LMI conditions given below

$$
M\left(R_{i}, S_{i}^{1}, \ldots, S_{i}^{m}\right)-2 \lambda_{i} N\left(R_{i}\right)<0, \quad i \in \overline{\mathrm{p}}
$$

The matrices $M$ and $N$ in the left side of the above inequality are given by

$$
M\left(R_{i}, S_{i}^{1}, \ldots, S_{i}^{m}\right):=\mathbf{A}_{i}{ }^{\prime} R_{i} \mathbf{E}_{i}+\mathbf{E}_{i}{ }^{\prime} R_{i} \mathbf{A}_{i}+\operatorname{diag}\left\{\sum_{j=1}^{m} S_{i}^{j},-e^{-2 \lambda_{i} h_{i}^{1}} S_{i}^{1}, \ldots,-e^{-2 \lambda_{i} h_{i}^{m}} S_{i}^{m}\right\}
$$

and

$$
N\left(R_{i}\right):=\operatorname{diag}\left\{R_{i}, 0_{q_{i}}, \ldots, 0_{q_{i}}\right\}
$$

where

$$
\mathbf{A}_{i}=\left[\begin{array}{llll}
\tilde{A}_{i}^{0}+\tilde{B}_{i} \tilde{K}_{i} \tilde{C}_{i} & \tilde{A}_{i}^{1} & \cdots & \tilde{A}_{i}^{m}
\end{array}\right], \quad \mathbf{E}_{i}=\left[\begin{array}{llll}
I_{q_{i}} & 0_{q_{i}} & \cdots & 0_{q_{i}}
\end{array}\right]
$$

3) Use the results of Lemma 4 and $\sigma_{i}$ given in step 2, find $\alpha_{i}$.

\section{Switching Algorithm}

It is desired now to develop a switching control strategy. Suppose that the constants $\alpha_{j}, \beta_{j}, \sigma_{j}, \lambda_{j}, \gamma_{j_{1}}, \gamma_{j_{2}}$ and $\gamma_{j_{3}}, j \in \overline{\mathrm{p}}$, are all chosen such that Lemmas 2 and 3 both hold. Assume also that $h:=\max \left\{h_{1}, \ldots, h_{p}\right\}$. The proposed switching scheme consists of two phases.

Phase 1: Finding the bound on the initial function. It is assumed that $\tilde{u}(t)=0$ for $t \in[0, T]$ for any arbitrary positive (nonzero) $T$ and, $z(0)=0$. Define

$$
\rho_{j}:=\sup _{-h_{j} \leq r<0} \frac{1}{\eta_{j}(r)}\left\|\int_{0}^{T} \Theta_{j}^{\prime}(t, r) C_{j}^{\prime} y(t) d t\right\|
$$

where $j \in \overline{\mathrm{p}}$. Suppose that $\|\omega(t)\| \leq \bar{b}$, and let the following auxiliary signals for $t \in[0, T]$ be defined

$$
\dot{r}_{j}=\lambda_{j} r_{j}(t)+\gamma_{j_{2}}\left\|\tilde{K}_{j}\left(\tilde{y}(t)-\tilde{D}_{j} y_{r e f}(t)\right)\right\|+\gamma_{j_{3}} \bar{b}
$$

with the initial condition $r_{j}(0)=0$. Define also

$$
\mu_{j}:=\rho_{j}+\beta_{j} \bar{b}
$$


If the plant is $\mathrm{P}_{j}$, then it follows from Lemma 2 that

Phase 2: Searching the gains. Let the control input be

$$
\max _{-h_{j} \leq r \leq 0}\|\tilde{\phi}(r)\| \leq \mu_{j}
$$

$$
\tilde{u}(t)=\tilde{K}_{i} \tilde{y}(t), \quad t \in\left(t_{i}, t_{i+1}\right]
$$

Consider the following $p$ auxiliary signals

$$
\dot{r}_{j}(t)=\lambda_{j} r_{j}(t)+\gamma_{j_{2}}\left\|\tilde{u}(t)-\tilde{K}_{j}\left(\tilde{y}(t)-\tilde{D}_{j} y_{r e f}(t)\right)\right\|+\gamma_{j_{3}} \bar{b}
$$

with $r_{j}\left(T^{+}\right)=r_{j}(T)+\gamma_{j_{1}} e^{\lambda_{j} T} \mu_{j}, j \in \overline{\mathrm{p}}$, and let the filtered signal be given by

$$
\dot{\tilde{r}}(t)=\tilde{\lambda} \tilde{r}(t)+(\lambda-\tilde{\lambda})\left\|\tilde{y}(t)-\tilde{D} y_{r e f}(t)\right\|, \quad \tilde{r}(T)=0
$$

where $\lambda:=\min \left\{\lambda_{i}: i \in \overline{\mathrm{p}}\right\}$ and $\tilde{\lambda}<\lambda$. It is to be noted that since the matrix $D_{j}$ is considered the same for all plant models, it is simply denoted by $\tilde{D}$ in the above equation. $r_{j}(t)$ gives an upper bound on the norm of the state for $t \geq T$ when the plant is $\mathrm{P}_{j}$. Moreover, $\tilde{r}$ filters $\tilde{y}-\tilde{D} y_{\text {ref }}$ to obtain a smooth error signal. Therefore, the switching instants are recursively defined as follow: set $t_{1}:=T$, and for any $i \in\{2, \ldots, p+1\}$ define $t_{i}$ as

$$
\min \left\{t \geq t_{i-1} \mid \text { there exist a time } \tilde{t} \in[T, t] \text {, for which } \tilde{r}(\tilde{t})=\left\|\tilde{C}_{i-1}\right\| r_{i-1}(\tilde{t})+\left\|\tilde{F}_{i-1}\right\| \tilde{b}+\varepsilon\right\}
$$

where $\varepsilon$ is any arbitrary (small) positive number.

\section{Properties of the Proposed Switching Controller}

Lemma 5: Suppose that $\tilde{\lambda}<\lambda \leq \lambda_{j}<0, \gamma_{1} \geq 0, \gamma_{2} \geq 0, \phi_{1}() \geq 0,. \phi_{2}() \geq$.0 ,

$$
\begin{aligned}
& \dot{v}_{1}(t)=\lambda_{j} v_{1}(t)+\phi_{1}(t), \quad v_{1}(0) \geq 0 \\
& \dot{v}_{2}(t)=\tilde{\lambda} v_{2}(t)+(\lambda-\tilde{\lambda}) \phi_{2}(t), \quad v_{2}(0)=0 .
\end{aligned}
$$

If $\phi_{2}(t) \leq \gamma_{1} v_{1}(t)+\gamma_{2}$ for $t \geq 0$, then $v_{2}(t) \leq \gamma_{1} v_{1}(t)+\gamma_{2}$ for $t \geq 0$ as well.

Theorem 1: Suppose that $y_{\text {ref }}(t)$ and $\omega(t)$ are bounded piecewise continuous signals, and that $\|\omega(t)\| \leq \bar{b}$ for $t \geq 0$. For any continuous initial function $\phi(r), r \in[-h, 0]$, the closed-loop system under the proposed switching algorithm has the following properties:

i) the gain eventually remains constant at an element of $\left\{\tilde{K}_{i}: i \in \overline{\mathrm{p}}\right\}$.

ii) the state $\tilde{x}(t)$ is bounded.

Proof: Let $y_{\text {ref }}(t), \omega(t)$ be piecewise continuous signals. Assume that $\|\omega(t)\| \leq \bar{b}$ for $t \geq 0$. Let $\phi(r)$ be any arbitrary initial function. Suppose now that the real plant is $\mathrm{P}_{j}, j \in \overline{\mathrm{p}}$. It follows from Lemma 2 and the definition of $\mu_{j}$ in (30), that $\max _{-h_{j} \leq r \leq 0}\|\tilde{\phi}(r)\| \leq \mu_{j}$. Now, using the result of Lemma 3

$$
\|\tilde{x}(t)\| \leq \gamma_{j_{1}} e^{\lambda_{j} t} \max _{-h \leq r \leq 0}\|\tilde{\phi}(r)\|+\int_{0}^{t} e^{\lambda_{j}(t-\tau)}\left[\gamma_{j_{2}}\left\|\tilde{u}(\tau)-\tilde{K}_{j}\left(\tilde{y}(\tau)-\tilde{D}_{j} y_{r e f}(\tau)\right)\right\|+\gamma_{j_{3}}\|\tilde{\omega}(\tau)\|\right] d \tau
$$

for $t>T$. Consequently,

$$
\|\tilde{x}(t)\| \leq \gamma_{j_{1}} \mu_{j} e^{\lambda_{j} t}+\int_{0}^{t} e^{\lambda_{j}(t-\tau)}\left[\gamma_{j_{2}}\left\|\tilde{u}(\tau)-\tilde{K}_{j}\left(\tilde{y}(\tau)-\tilde{D}_{j} y_{r e f}(\tau)\right)\right\|+\gamma_{j_{3}} \bar{b}\right] d \tau
$$

Thus, for $t>T$

$$
\|\tilde{x}(t)\| \leq r_{j}(t)
$$

and hence

$$
\left\|\tilde{y}(t)-\tilde{D}_{j} y_{r e f}(t)\right\| \leq\left\|\tilde{C}_{j}\right\| r_{j}(t)+\left\|\tilde{F}_{j}\right\| \tilde{b}
$$

It follows from Lemma 5 that for $t>T$,

$$
\tilde{r}(t) \leq\left\|\tilde{C}_{j}\right\| r_{j}(t)+\left\|\tilde{F}_{j}\right\| \tilde{b}
$$

Therefore, it can be concluded that the first property holds. It remains to show that $\tilde{x}(t)$ is bounded. From (31) it follows that one suffices to show that $r_{j}$ is bounded. Let the final gain be $\tilde{K}_{i}$. Since $y_{r e f}(t), \omega(t)$ are bounded piecewise signals, $r_{i}(t)$ is also bounded. This leads to the boundedness of $\tilde{r}(t)$. On the other hand, for $t \geq T$

$$
\int_{T}^{t} e^{\lambda_{j}(t-\tau)}(\lambda-\tilde{\lambda})\left\|\tilde{y}(\tau)-\tilde{D} y_{r e f}(\tau)\right\| d \tau=\tilde{r}(t)+\left(\lambda_{j}-\tilde{\lambda}\right) \int_{T}^{t} e^{\lambda_{j}(t-\tau)} \tilde{r}(\tau) d \tau
$$

Thus,

$$
\sup _{t>T} \int_{T}^{t} e^{\lambda_{j}(t-\tau)}\|\tilde{y}(\tau)\| d \tau<\infty
$$


This results in the boundedness of $r_{j}(t)$.

The following two assumptions will be used to achieve zero tracking error for a pre-specified set of reference inputs and disturbances.

Assumption 1: The entries of $y_{r e f}(t)$ and $\omega(t)$ are assumed to be described by the following differential equation

$$
(.)^{(v)}+\xi_{v-1}(.)^{(v-1)}+\ldots+\xi_{1}(.)^{(1)}+\xi_{0}(.)=0
$$

with independent initial conditions. Moreover, the roots of

$$
s^{v}+\xi_{v-1} s^{v-1}+\ldots+\xi_{1} s+\xi_{0}=0
$$

are assumed to be distinct and purely imaginary.

Assumption 2: For every $i, l \in \overline{\mathrm{p}}$, the matrix $\tilde{K}_{l}$ has the property that the roots of the following equation do not lie on the imaginary axis

$$
\Delta_{i}(s)=\operatorname{det}\left(s I-\tilde{A}_{i}^{0}-\tilde{B}_{i} \tilde{K}_{l} \tilde{C}_{i}-\sum_{j=1}^{m} \tilde{A}_{i}^{j} e^{-h_{i}^{j} s}\right)=0
$$

Condition of Assumption 2 implies that $\tilde{K}_{l}$ is designed such that the error signal, when the plant is $\tilde{\mathrm{P}}_{i}$, can be written in terms of strictly growing or strictly decaying exponentials and not sinusoids or constants.

Assumption 3: Suppose that controllers $\mathrm{K}_{i}$ are designed such that each of them solves the servomechanism problem for the corresponding plant $\mathrm{P}_{i}$, i.e. $\mathrm{K}_{i}$ is chosen in such a way that exact tracking of $y_{\text {ref }}$ is achieved despite the existence of external disturbances in the system. Similar to finite-dimensional case [31], one should first design a servo-compensator, which in fact augments the poles of (32) to the open loop system, and then try to find a stabilizing controller for the plant and servo-compensator combination. It can be shown for both finite and infinite-dimensional cases such a controller configuration has the property that the zeros of each entry in the transfer function from the reference and the disturbance to the error includes all the roots of (33).

Corollary 2: Consider the system (1) and suppose that the conditions of Assumptions 1, 2 and 3 hold. In addition, assume that $\|\omega(t)\| \leq \bar{b}$ for $t \geq 0$. Then, for any continuous initial function $\phi(r), r \in[-h, 0]$, the error signal $e(t)$ resulted by applying the proposed switching scheme approaches zero as $t \rightarrow \infty$.

Proof: Let $y_{\text {ref }}$ and $\omega$ be such that any of its elements satisfies (32). It is known from theorem 1 that the gain eventually remains constant and $\tilde{x}$ and $\tilde{y}$ are bounded. By Assumption 2, the final gain, say $\tilde{\mathrm{K}}_{i}$, is such that the final LTI closed loop system has no poles on the imaginary axis. In addition, from Assumption 3, it follows that $E(s)$ (the Laplace transform of $e(t)$ ) does not have any pole at each root of (33). Therefore, the error is a sum of weighted exponentials corresponding to poles of the closed loop system, none of which lie on the imaginary axis. Then, it follows from the boundedness of $e$ (which follows from the boundedness of $\tilde{y}$ ) that only the exponentials which decay to zero have non-zero weights. Hence, $e(t) \rightarrow 0$ as $t \rightarrow \infty$, as required.

Remark 4: It is to be noted that the system may lock onto a stabilizing controller, which is not necessarily the highperformance controller designed for the corresponding plant model. However, to avoid this problem, one may use an approach similar to [32] to design the controllers such that each one stabilizes only one of the plant models.

\section{NUMERICAL EXAMPLE}

Consider the following single-input single-output system

$$
\begin{aligned}
\dot{x}_{1}(t) & =x_{2}(t)+x_{2}\left(t-h_{0}\right)+u(t)+\omega(t) \\
\dot{x}_{2}(t) & =-2 x_{1}(t)-3 x_{2}(t) \\
y(t) & =c x_{1}(t)
\end{aligned}
$$

A family of three plant models is considered as follows

$$
\begin{array}{ll}
\mathrm{P}_{1}: & h_{0}=0.1, c=1 \\
\mathrm{P}_{2}: & h_{0}=0.6, c=1 \\
\mathrm{P}_{3}: & h_{0}=0.1, c=-1
\end{array}
$$

$y_{\text {ref }}$ is assumed to be a square wave of magnitude 1 and period $4 \mathrm{sec}$. Let the following PI controllers be used to achieve reference tracking and disturbance rejection

$$
\begin{array}{ll}
\mathrm{K}_{1}: & \dot{z}=e, u=12 z+6 e \\
\mathrm{~K}_{2}: & \dot{z}=e, u=16 z+8 e \\
\mathrm{~K}_{3}: & \dot{z}=e, u=-12 z-6 e
\end{array}
$$




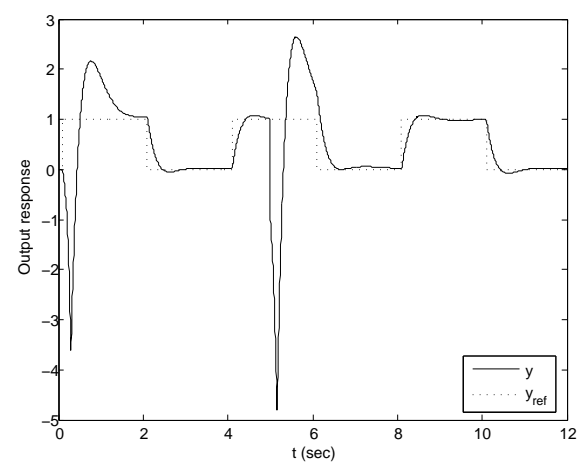

Fig. 1. Output response for the system (34), using the proposed switching scheme.

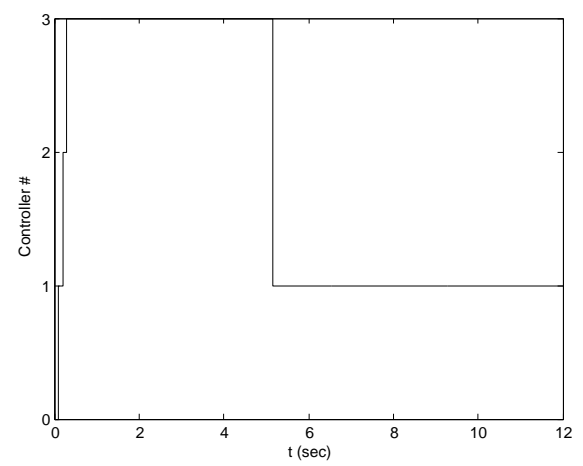

Fig. 2. Switching control sequence for the numerical example.

Suppose that $\omega(t)=0$, and choose $T$ in phase 1 equal to $0.1 \mathrm{sec}$. This implies that for $t \in[0,0.1]$ (i.e., during phase 1 ), the control signal applied to the system is identical to zero, and immediately after that different controllers are examined. The system will first switch to the controller $\mathrm{K}_{1}$. Since this is not the stabilizing controller for $\mathrm{P}_{3}$, the error will hit the upper bound signal and thus, the system will switch to the controller $\mathrm{K}_{2}$. This will also destabilize the plant and eventually at time $0.29 \mathrm{sec}$, the system will switch to the stabilizing controller $\mathrm{K}_{3}$. Figure 1 depicts the output response of the system and Figure 2 gives the switching sequence. It is to be noted that in the above switching sequence, the plant will examine two destabilizing controllers $\mathrm{K}_{1}$ and $\mathrm{K}_{2}$ but as it can be seen from Figure 1, the resultant transient magnitude is about 3.5 which is good.

Assume now that at $t=5 \mathrm{sec}$, the plant changes from $\mathrm{P}_{3}$ to $\mathrm{P}_{1}$. As a result, the error will hit its corresponding upper bound signal in about $0.15 \mathrm{sec}$ and the system will then switch to $\mathrm{K}_{1}$, which is the stabilizing controller for $\mathrm{P}_{1}$. It is to be noted that one of the shortcomings of most switching control schemes is the large magnitude of the transient response. One can use the multi-layer switching mechanism introduced in [33] to improve the transient response.

\section{CONCLUSIONS}

In this paper, adaptive switching control of retarded time-delay LTI systems is considered. A switching control scheme is proposed to stabilize and regulate the system, which is an extension of the method introduced by [8] for finite dimensional LTI systems. In this switching control scheme, it is assumed that the plant can be described by a family of retarded timedelay LTI models, and that a set of high-performance controllers has been obtained, so that the actual plant model can be stabilized and regulated by at least one controller contained in this set. The simulation results demonstrate the effectiveness of the proposed scheme.

\section{REFERENCES}

[1] D. R. Mudgett and A. S. Morse, "Adaptive stabilization of linear systems with unknown high frequency gains," IEEE Trans. on Automat. Contr., vol. 30, no. 6 , pp. 549-554, Jun. 1985.

[2] J. C. Willems and C. I. Byrnes, "Global adaptive stabilization in the absence of information on the sign of the high frequency gain," In A. Bensoussan and J. L. Lions (Eds), Analysis and Optimization of Systems: Proc. of the Sixth Int. Conf. on Anal. and Opt. of Syst., vol. 62 of Lecture Notes in Control and Information Sciences, Springer-Verlag, Berlin, pp. 49-57, 1984.

[3] T. H. Lee and K. S. Narendra, "Stable discrete adaptive control with unknown high-frequency gain," IEEE Trans. on Automat. Contr., vol. 31 , no. 5 , pp. 477-479, May 1986. 
[4] A. S. Morse, "A 4(n+1)-dimensional model reference adaptive stabilizer for any relative degree one or two, minimum phase system of dimension $n$ or less," Automatica, vol. 23, no. 1, pp. 123-125, Jan. 1987.

[5] B. Mårtensson, "The order of any stabilizing regulator is sufficient a priori information for adaptive stabilization," Syst. \& Contr. Lett., vol. 6, no. 2, pp. 87-91, Feb. 1985

[6] D. E. Miller and E. J. Davison, "On necessary assumption in continuous time model reference adaptive control," Proc. of the 28th IEEE Conf. on Decision and Contr., pp. 1573-1578, 1989.

[7] A. S. Morse, "Recent problems in parameter adaptive control," Proc. CNRS Colloquium on Development and Utilization of Mathematical Models in Automat. Contr., Belle-Isle, France, pp. 733-740, Sept. 1982.

[8] D. E. Miller and E. J. Davison, ”Adaptive control of a family of plants," In D. Hinrichsen, B. Mårtensson (Eds.), Control of Uncertain Systems: Proc. of an Int. Workshop, Bermen, West Germany, Jun. 1989, Progress in Systems and Control Theory, vol. 6, pp. 197-219, Birkhäusser Press, Boston, 1990.

[9] D. E. Miller and E. J. Davison, "An adaptive tracking problem," Int. Journal of Adaptive Contr. and Signal Processing, vol. 6, no. 1, pp. 45-63, Jan. 1992.

[10] A. S. Morse, "Supervisory control of families of linear set-point controllerspart 1: exact matching," IEEE Trans. on Automat. Contr., vol. 41, no. 10, pp. 1413-1431, Oct. 1996.

[11] K. S. Narendra and J. Balakrishnan, ”Adaptive control using multiple models," IEEE Trans. on Automat. Contr., vol. 42, no. 2, pp. 171-187, Feb. 1997.

[12] M. Chang and E. J. Davison, "Adaptive switching control of LTI MIMO systems using a Family of controllers approach," Automatica, vol. 35, no. 3, pp. 453-465, Mar. 1999.

[13] D. Angeli and E. Mosca, "Lyapunov-based switching supervisory control of nonlinear uncertain systems," IEEE Trans. on Automat. Contr., vol. 47, no. 3, pp. 500-505, Mar. 2002.

[14] A. G. Aghdam and E. J. Davison, ”Pseudo-decentralized switching control," Automatica, vol. 39, no. 2, pp. 317-324, Feb. 2003.

[15] M. Fu and B. R. Barmish, "Adaptive stabilization of linear systems via switching control," IEEE Trans. on Automat. Contr., vol. 31, no. 12, pp. 1097-1103, Dec. 1986.

[16] D. E. Miller and E. J. Davison "An adaptive controller which provides lyapunov stability," IEEE Trans. on Automat. Contr., vol. 34, no. 6, pp. 599-609, Jun. 1989.

[17] K. S. Narendra and J. Balakrishnan, "Improving transient response of adaptive control systems using multiple models and switching," IEEE Trans. on Automat. Contr., vol. 39, no. 9, pp. 1861-1866, Sept. 1994.

[18] Joao Hespanha, Tutorial on supervisory control, Lecture Notes for the workshop Control using Logic and Switching for the 40th IEEE Conf. on Decision and Contr., Orlando, Florida, Dec. 2001.

[19] D. E. Miller and D. E. Davison, "Stabilization in the presence of an uncertain arbitrarily large delay," IEEE Trans. on Automat. Contr., vol. 50, no. 8, pp. 1074-1089, Aug. 2005.

[20] C. Lin, Q. Wang and T. H. Lee, "A less conservative robust stability test for linear uncertain time-delay systems," IEEE Trans. on Automat. Contr. vol. 51, no. 1, pp. 87-91, Jan. 2006.

[21] K. Shyu, W. Liu and K. Hsu, ”Design of large-scale time-delayed systems with dead-zone input via variable structure control," Automatica, vol. 41, no. 7, pp. 1239-1246, Jul. 2005.

[22] K. Gu, L. Kharitonov and J. Chen, Stability of time-delay systems, Birkhauser, Boston, 2003.

[23] M. Bozorg and E. J. Davison, "Control of time delay processes with uncertain delays: time delay stability margins," Journal of Process Contr, vol. 16, no. 4, pp. 403-408, Apr. 2006.

[24] E. Fridman, "Output regulation of nonlinear systems with delay" Syst. \& Contr. Lett., vol. 50, no. 2, pp. 81-93, Oct. 2003

[25] J. K. Hale and S. M. V. Lunel, Introduction to functional differntial equations, Springer-Verlag, N.Y., 1993.

[26] V. L. Kharitonov and D. Hinrichsen, "Exponential estimates for time delay systems," Syst. \& Contr. Lett., vol. 53, no. 5, pp. 395-405, Dec. 2004.

[27] K. Bhat and H. Kovio, "Modal characterizations of controllability and observability in time delay systems," IEEE Trans. on Automat. Contr., vol. 21, no. 2, pp. 292-293, Apr. 1976.

[28] A. Olbrot, "A counterexample to observability of linear systems with time-variable delays," IEEE Trans. on Automat. Contr., vol. 22, no. 2, pp. 281-283, Apr. 1977.

[29] C. Van Loan, "Computing integrals involving the matrix exponential," IEEE Trans. on Automat. Contr., vol. 23, no.3, pp. 395-404, Jun. 1978.

[30] S. Mondie and V. L. Kharitonov, "Exponential estimates for retarded time-Delay systems: an LMI approch," IEEE Trans. on Automat. Contr., vol. 50, no. 2, pp. 268-273, Feb. 2005.

[31] E. J. Davison, "A generalization of the output control of linear multivariable systems with unmeasurable arbitrary disturbances," IEEE Trans. on Automat. Contr., vol. 20, no. 6, pp. 788-792, Dec. 1975.

[32] M. Athans, S. Fekri and A. Pascoal, "Issues on robust adaptive feedback control," Proc. of the 16th IFAC World Congress, Prague, Czech Republic, Jul. 2005.

[33] S. Zahirazami, I. Karuei and A. G. Aghdam, "Multi-layer switching structure with periodic feedback control," Proc. American Contr. Conf., pp. $5425-5431,2006$.

[34] S. Zahirazami, I. Karuei and A. G. Aghdam, "Multi-layer switching control using generalized sampled-data hold functions," accepted for publication in European Journal of Control. 